\title{
Recent Observations of GRB-Supernovae
}

\author{
Bethany Elisa Cobb \\ George Washington University \\ Department of Physics \\ 725 21st St, NW, Corcoran 105 \\ Washington DC, 20052, USA \\ email: bcobb@gwu.edu
}

\begin{abstract}
The GRB-SNe connection has been strengthened since 2008 by the detection of 6 additional GRB-SNe at both local and cosmological redshifts. This review summarizes the recent observations of SNe associated with GRBs 081007, 090618, 091127, 100316D, 101219B and $111211 \mathrm{~A}$, as well as the observations of SN 2008D, which was associated with a bright X-ray flash (XRF 080109) and may represent a link between "plain" SN and GRB-SNe. It is now clear that most - if not all - long-duration GRBs are produced by the core collapse of massive stars.
\end{abstract}

Keywords. gamma rays: bursts, supernovae: general, supernovae: individual (2008D, 2008hw, 2009nz, 2010bh, 2010ma)

\section{Introduction}

The connection between long-duration gamma-ray bursts (GRBs) and supernovae $(\mathrm{SNe})$ is supported by indirect evidence including energy considerations (e.g. Woosley 1993), the association of long-duration GRBs with star-forming host galaxies (e.g. Djorgovski et al. 1998), and the similarity between the environments in which GRBs occur and the environments in which "plain" SNe-Ic occur (Raskin et al. 2008). Between 1998 and 2006, a number of GRBs were also directly connected - both photometrically and spectroscopically - with SNe, including the prime examples of GRB 980425/SN 1998bw, GRB 030329/SN 2003dh, GRB 031203/SN 2003lw, GRB 050525A/SN 2005nc and GRB 060218/2006aj (see Table 1, see also Woosley \& Bloom 2006). Interestingly, three of these early, well-studied GRB-SNe (GRBs 980425, 031203 and 060218) had low redshifts $(z \lesssim 0.1)$ and low gamma-ray luminosities (isotropic energy $E_{\text {iso }} \sim 10^{48-50} \mathrm{erg}$, 2 to 4 orders of magnitude lower than typical for GRBs observed at cosmological distances), showed divergence from the $E_{\text {peak }}-E_{i s o}$ correlation (Amati relation, e.g. Amati 2006) and produced extremely faint or undetected optical afterglows. Questions naturally arose as to whether or not this group of GRB-SNe represented a special, local population or if all long-duration GRBs are associated with SNe. The detection of additional GRB$\mathrm{SNe}$, therefore, is crucial in the test of whether or not the GRB-SN connection holds in general for all GRBs, including those with high-redshift and high luminosity.

This review summarizes the recent (since 2008) observations of GRB-related SNe.

\section{XRF $080109-$ SN 2008D at $z=0.007$ : a special non-GRB SN}

The first SN is not actually a GRB-SN, but may represent a link in the continuum between "normal" SNe and GRB-SNe, and therefore merits discussion.

The Swift X-ray Telescope (XRT; Burrows et al. 2005) serendipitously detected an X-ray flash/transient in NGC 2770 on 2008 January 9 at 13:32:49 UT (Soderberg et al. 2008b). This transient does not appear to have been produced by a GRB, as Swift 
(Gehrels et al. 2004) detected no gamma-ray emission from the source despite the fact that the XRF was within the field of view of the Burst Alert Telescope (BAT; Barthelmy et al. 2005). While an optical counterpart was not seen in contemporaneous Ultraviolet/Optical Telescope (UVOT; Roming et al. 2005) imaging, a brightening counterpart was detected just 1.4 hours after the flash occurred. This bright X-ray flash $\left(E_{X} \sim 10^{46} \mathrm{erg}\right)$ was quickly recognized to have been produced by the core collapse of a massive star. Optical imaging and spectra revealed a Helium-rich type Ib/c SN (Deng \& Zhu 2008, Soderberg et al. 2008a).

The peak optical luminosity of the event fell on the low end of the distribution of GRBSNe luminosities, but the SN was very similar to other GRB-SNe in having a rest-frame rise time ( 18 days) longer than typical for "plain" SN Ib/c (Modjaz et al. 2009). In contrast, the spectral features of this SN were narrower than observed in other GRB-SNe (Malesani et al. 2008).

The remarkable photometric and spectroscopic temporal coverage of this event allowed for detailed modeling of the SN and its progenitor. Interestingly, the SN appears to have synthesized $\sim 0.09 M_{\odot}$ of ${ }^{56} \mathrm{Ni}$, an amount similar to that seen in the broad-lined SN-Ic 2002ap, but much less than that seen in most GRB-SNe, including SN 1998bw which synthesized $\sim 0.7 M_{\odot}$ of ${ }^{56} \mathrm{Ni}$ (Tanaka et al. 2009).

The X-ray flash of SN 2008D may be attributed to a shock break-out from a WolfRayet star, which was slightly delayed by the presence of a dense stellar wind around the progenitor (Soderberg et al. 2008b). Alternatively, the X-ray flash may result from the collapse of $\mathrm{a} \sim 30 M_{\odot}$ star that produced a black hole with a weak, mildly relativistic jet (Mazzali et al. 2008). In the latter case, the jet is a "failed" relativistic jet - likely failing due to low initial energy or to damping caused by a helium layer, which would not be found around most pre-GRB stars (Mazzali et al. 2008). This SN, therefore, may indicate that GRB-like central engine activity may be present in all black-hole-forming SNe Ibc. Even if this SN did not produce a mildly relativistic jet, it may still be an example of a link between He-rich and He-poor explosions (a class which includes the GRB-SNe).

\section{GRB $081007-\mathrm{SN} 2008 \mathrm{hw}$ at $z=0.53$}

Swift triggered on the long-duration GRB 081007 on 2008 October 7 at 05:23:52 UT (Baumgartner et al. 2008). The Fermi Gamma-ray Burst Monitor (GBM) also triggered on this GRB (Bissaldi et al. 2008). The GRB had a typical isotropic energy release of $E_{i s o} \sim 10^{51} \mathrm{erg}$. The burst's X-ray afterglow was detected by the XRT and the burst's optical afterglow was detected by UVOT. Ground-based imaging by the robotic $60-\mathrm{cm}$ Rapid Eye Mount (REM; Chincarini et al. 2003) telescope also identified the burst's bright, slowly fading optical/NIR afterglow (Covino et al. 2008). Only 73 minutes postburst, a Gemini Multi-Object Spectrograph (GMOS) observation was started and the redshift of the burst was determined to be $z=0.53$ (Berger et al. 2008).

The SN associated with GRB 081007, SN 2008hw, was first noticed in Gemini-GMOS imaging as an increase in optical afterglow brightness detected between about 17 and 26 days using image subtraction techniques (Soderberg et al. 2008c). Analysis of a Very Large Telescope (VLT) spectrum taken 17 days post-burst then revealed (following the subtraction of a template starburst galaxy) the broad bumps indicative of a GRB-SN similar to SN 1998bw (Della Valle et al. 2008). Additional photometric follow-up carried out by the Gamma-Ray Burst Optical/Near-Infrared Detector (GROND; Greiner et al. 2008) showed a flattening of the afterglow light curve at approximately 7 days (restframe) post-burst and a change in the spectral-energy distribution of the afterglow at 10 
days (rest-frame) post-burst, consistent with the increased contribution of light from a brightening SN (Della Valle et al. 2008).

Of the recent GRB-SNe, GRB 081007/SN 2008hw serves as just the first example of a "typical" energy GRB with a "typical" optical afterglow that is located at "cosmological" distances and yet is undeniably associated with a SN.

\section{GRB 090618 - SN2009?? at $z=0.54$}

Swift triggered on the long-duration GRB 090618 on 2009 June 18 at 08:28:29 UT (Schady et al. 2009). This was an extremely bright burst that also triggered Fermi-GBM (McBreen 2009), Super-AGILE (Longo et al. 2009), and Konus-Wind (Golenetskii et al. 2009). The burst's X-ray afterglow was detected by the XRT and the burst's optical afterglow was detected by UVOT. Numerous ground-based telescopes also observed this burst's bright optical afterglow including the Palomar 60-inch (Cenko 2009), the Katzman Automatic Imaging Telescope (KAIT; Perley 2009) and the Robotic Optical Transient Search Experiment (ROTSE)-IIIb (Rujopakarn et al. 2009). Just 20 minutes after the burst, the Kast spectrograph on the 3-m Shane telescope at Lick Observatory began obtaining observations, and the redshift of the burst was determined to be $z=0.54$ (Cenko et al. 2009).

The SN associated with GRB 090618 was identified by the presence of a "bump" in the optical afterglow light curve, which was associated with a change in the afterglow's $R_{c}-i$ color index. The color change is expected to occur as the early, blue afterglow fades and the later, redder SN component rises. The SN accompanying GRB 090618 is similar in brightness and temporal evolution to the prototypical GRB-SN, SN 1998bw. After the host galaxy and afterglow contributions were subtracted, it was determined that the SN peaked around 16 days (rest-frame) and was slightly brighter than SN 1998bw (Cano et al. 2011).

GRB 090618 had "typical" gamma-ray burst parameters, including an isotropic equivalent energy release of $E_{i s o} \sim 3 \times 10^{53} \mathrm{erg}$ (Page et al. 2011, Izzo et al. 2012). GRB 090618 is, therefore, an excellent example of a normal, cosmological GRB associated with a SN. Unfortunately, no spectrographic observations of this GRB-SN were obtained.

\section{GRB $091127-$ SN 2009nz at $z=0.49$}

Swift triggered on the long-duration GRB 09117 on 2009 November 27 at 23:25:45 UT (Troja et al. 2009). This burst also triggered Fermi-GBM (Wilson-Hodge \& Preece 2009) and the Suzaku Wide-band All-sky Monitor (WAM; Nishioka et al. 2009). While no XRT or UVOT observations were initially obtained due to observing constraints, the robotic ground-based 2-m Liverpool Telescope detected a bright $(R \sim 15)$ optical afterglow just minutes after the burst occurred (Smith et al. 2009). An X-ray afterglow was eventually detected by XRT (Evans et al. 2009). The optical afterglow was bright enough to be detected by numerous instruments including UVOT (Immler \& Troja 2009), REM (Fugazza et al. 2009), GROND (Updike et al. 2009), and the $1.3 \mathrm{~m}$ Small and Moderate Aperture Research Telescope System (SMARTS; Subasavage et al. 2010) telescope (Cobb 2009). The redshift of the burst was measured to be $z=0.49$ by a Gemini-GMOS spectroscopic observation (Cucchiara et al. 2009) and confirmed by an X-shooter (D'Odorico et al. 2006) observation at the VLT (Thoene et al. 2009).

The SN was detected as a flattening then a brightening of the optical afterglow in a combination of Gemini-GMOS and 1.3m SMARTS imaging. The optical afterglow began to brighten around 10 days (observer frame) post-burst, following an evolution similar to 
SN 1998bw (Cobb et al. 2010a). The SN was designated SN 2009nz (Cobb et al. 2010b). Comparison of SN 2009nz to other well-observed GRB-SNe shows that these events have global similarities, but each one shows individual variations in peak brightness and risetime (Cobb et al. 2010c). While the GRB-SNe form a relatively homogenous group, they are certainly not "clones" of one another.

Spectral identification of SN 2009nz was later published, revealing the broad features typical of GRB-SNe due to their high expansion velocities. Uncovering the SN spectrum required taking a late-time ( $\sim 1$ year post-burst) spectrum of the host galaxy. This was used to subtract the host's contribution from a spectrum taken near the SN's peak brightness. The spectrum of SN 2009nz was reminiscent of SN 2006aj, and had narrower features than SN 1998bw and SN 2010bh, indicating a somewhat lower expansion velocity (Berger et al. 2011).

GRB 091127 had "typical" GRB parameters, including an $E_{i s o} \sim 10^{53} \mathrm{erg}$ (Stamatikos et al. 2009), providing another example of a link between a cosmological long-duration GRB with standard energy to the core-collapse of a massive star. This burst also highlights the difficulty of detecting cosmological GRB-SNe, since both the photometric and spectroscopic observations of this GRB-SN were complicated by the burst's relatively high redshift and light contamination from both the GRB's bright optical afterglow and its relatively bright host galaxy.

\section{GRB 100316D - SN 2010bh at $z=0.059$}

Swift triggered on the long-duration GRB 100316D on 2010 March 16 at 12:44:50 UT (Stamatikos et al. 2010). An X-ray afterglow was detected, but no UVOT source was observed (Stamatikos et al. 2010). An optical source inside the XRT error region was detected in a VLT/X-shooter acquisition image, with a measured redshift of $z=0.059$ (Vergani et al. 2010a). Initially it was unclear whether or not this optical source was associated with the GRB or if it was an extended region of a nearby DSS galaxy. GeminiGMOS imaging combined with further VLT/X-shooter spectroscopy confirmed that the optical source and the nearby galaxy were at a common redshift and thus likely two parts of a single, morphologically disturbed galaxy (Vergani et al. 2010b).

With an isotropic energy release of only $4-6 \times 10^{49} \mathrm{erg}$, a redshift of only $z=$ 0.059 , and an extremely long duration, this burst was not a typically "cosmological" GRB, but was reminiscent of the group of low-redshift, low gamma-ray luminosity bursts detected prior to 2006 (GRBs 980425, 031203 and 060218). Similar to those events, no optical afterglow was detected following the GRB, though the presence of the bright galaxy at the position of the X-ray afterglow may easily have obscured a dim optical afterglow. GRB 100316D shared temporal characteristics with GRB 060218/SN 2006aj; both of these bursts also had very soft gamma-ray spectra (Sakamoto et al. 2010a). The notable similarities between these two bursts suggested that observers should be on the lookout for an emergent SN (Sakamoto et al. 2010b). In Gemini-GMOS imaging acquired approximately 1.5 days post-burst, a possibly brightening source was noted (Levan et al. 2010). This brightening was eventually confirmed by additional Gemini-GMOS imaging (Wiersema et al. 2010) and imaging by GROND (Rau et al. 2010), indicating a rising SN associated with GRB 100316D: SN 2010bh (Bufano et al. 2010a). Spectroscopy obtained on the twin 6.5-m Magellan telescopes and VLT X-shooter and FORS2 then revealed broad photospheric absorption lines similar to SN 1998bw (Chornock et al. 2010a, Bufano et al. 2010b).

SN 100316D rose to peak brightness in approximately 8 days, which is faster than SN 1998bw but similar to SN 2006aj (Bufano et al. 2011, Starling et al. 2011). However, SN 
100316D showed higher photospheric velocities than SN 1998bw, possibly indicating that SN 100316D was a more energetic explosion than SN 1998bw (Chornock et al. 2010b). SN 2010bh is also the faintest of the well-studied GRB-SNe, which suggests it ejected smaller amounts of ${ }^{56} \mathrm{Ni}$ than the other GRB-SNe (Bufano et al. 2011).

\section{GRB 101219B - SN 2010ma at $z=0.55$}

Swift triggered on the long-duration GRB 101219B on 2010 December 19 at 16:27:53 UT (Gelbord et al. 2010). This GRB also triggered Fermi-GBM (van der Horst 2010). Swift detected both an X-ray and optical afterglow (Gelbord et al. 2010); the optical/NIR afterglow was also detected by GROND (Olivares et al. 2010). This is another example of a typical energy $\left(\sim 4 \times 10^{51} \mathrm{erg}\right)$ GRB with a bright afterglow that obeys the "Amati" relation (Sparre et al. 2011). The afterglow of this burst was particularly notable in that it had an unusually slow decay and was still detectable by UVOT at 2 weeks post-burst (Kuin \& Gelbord 2011).

The first hints of a SN appeared 5 days post-burst when the GROND afterglow light curve began to flatten and change color; at 15 days post-burst, the afterglow resumed its decay (Olivares et al. 2011). Several spectra obtained using VLT/X-shooter around 16 days post-burst measured a redshift of the optical source to be $z=0.55$, with the spectra showing broad SN-like undulations similar to those detected in SN 1998bw and all other GRB-SNe (de Ugarte Postigo et al. 2011).

\section{GRB 111211A - SN2011?? at $z=0.478$}

Super-AGILE (Tavani et al. 2009) triggered on the long-duration GRB 111211A on 2011 December 11 at 22:17:33 UT (Lazzarotto et al. 2011). GROND ground-based imaging identified the burst's optical afterglow approximately 8.5 hours post-burst (Kann et al. 2011). VLT/X-shooter spectroscopy obtained 1.3 days after the GRB measured the redshift of the burst to be $z=0.478$ (Vergani et al. 2011).

The SN associated with GRB 111211A was first detected in a spectrum obtained using the $10.4 \mathrm{~m}$ Gran Telescopio Canarias (GTC) equipped with the Ohio State Infrared Imager/Spectrometer (OSIRIS). The spectrum showed that at the position of the optical afterglow, there was a broad-line SN similar to SN 2006aj (associated with GRB 060218) close to its time of maximum (de Ugarte Postigo et al. 2012). Additionally, photometric comparison of the spectrum acquisition image to an r-band image taken 5 days prior showed that the afterglow's light curve had flattened, indicating a possible contribution from a supernova component.

\section{Conclusion}

These recent GRB-SNe observations have significantly added to the "GRB-SNe zoo" (see Table 1). No longer are GRB-SNe just associated with special cases of low-redshift, low-luminosity GRBs. By identifying additional examples of photometrically and spectroscopically confirmed GRB-SNe, it now appears that the GRN-SN connection is proven both for local, low-luminosity GRBs and for typical-energy, cosmological GRBs. Clearly, the evidence suggests that most - if not all - long-duration GRBs are intimately linked with SNe.

Furthermore, the special example of XRF 080109/SN 2008D, while not itself a GRBSN, might indicate a link between GRB-SNe and non-GRB-SNe. This event helps fill out the continuum between "typical" SNe and the "hyper" SNe associated with GRBs. 
Table 1. GRB/SNe

\begin{tabular}{rrr}
\hline GRB & SN & Redshift \\
\hline 980425 & $1998 \mathrm{bw}$ & 0.0085 \\
\hline 030329 & $2003 \mathrm{dh}$ & 0.16 \\
\hline 031203 & $2003 \mathrm{lw}$ & 0.11 \\
\hline $050525 \mathrm{~A}$ & $2005 \mathrm{nc}$ & 0.606 \\
\hline 060218 & $2006 \mathrm{aj}$ & 0.033 \\
\hline XRF 080109 & $2008 \mathrm{D}$ & 0.007 \\
\hline 081007 & $2008 \mathrm{hw}$ & 0.53 \\
\hline 090618 & $2009 ? ?$ & 0.54 \\
\hline 091127 & $2009 \mathrm{nz}$ & 0.49 \\
\hline $100316 \mathrm{D}$ & $2010 \mathrm{bh}$ & 0.059 \\
\hline $101219 \mathrm{~B}$ & $2010 \mathrm{ma}$ & 0.55 \\
\hline $111211 \mathrm{~A}$ & $2011 ? ?$ & 0.478 \\
\hline
\end{tabular}

Of course, questions about the GRB-SN connection still remain thanks to two examples of low-redshift GRBs (060505 \& 060614) without detected SNe (e.g. Fynbo et al. 2006). Possibly the redshifts of these bursts are mis-identified due to the chance alignment of a higher-redshift GRB with a foreground galaxy (e.g. Cobb et al. 2006). Alternatively, these bursts might by short-duration bursts and thus not produced by the core collapse of a massive star (e.g. Gehrels et al. 2006). However, it is also possible that something about the progenitors of these GRBs was unique and as yet unknown. In any case, it has now become clear that these examples are outliers, and that long-duration GRBs of all redshifts and energies are primarily produced by the core collapse of massive stars.

\section{References}

Amati, L. 2006, MNRAS, 372, 233

Barthelmy, S. D., et al. 2005, Space Sci. Revs, 121, 143

Baumgartner, W. H., et al. 2008, GCN, 8330, 1

Berger, E., Fox, D. B., Cucchiara, A., \& Cenko, S. B. 2008, GCN, 8335, 1

Berger, E., et al. 2011, ApJ, 743, 204

Bissaldi, E., McBreen, S., \& Connaughton, V. 2008, GCN, 8369, 1

Bufano, F., et al. 2010, CBET, 2227, 1

Bufano, F., et al. 2010, GCN, 10543, 1

Bufano, F., et al. 2011, arXiv, arXiv:1111.4527

Burrows, D. N., et al. 2005, Space Sci. Revs, 121, 165

Cano, Z., et al. 2011, MNRAS, 413, 669

Cenko, S. B. 2009, GCN, 9513, 1

Cenko, S. B., Perley, D. A., Junkkarinen, V., Burbidge, M., Diego, U. S., \& Miller, K. 2009, GCN, 9518, 1

Chincarini, G., et al. 2003, Messenger, 113, 40

Chornock, R., Soderberg, A. M., Foley, R. J., Berger, E., Frebel, A., Challis, P., Simon, J. D., \& Sheppard, S. 2010, GCN, 10541, 1

Chornock, R., et al. 2010, arXiv, arXiv:1004.2262

Cobb, B. E., Bailyn, C. D., van Dokkum, P. G., \& Natarajan, P. 2006, ApJ, 651, L85

Cobb, B. E. 2009, GCN, 10244, 1

Cobb, B. E., Bloom, J. S., Cenko, S. B., \& Perley, D. A. 2010, GCN, 10400, 1 
Cobb, B. E., Bloom, J. S., Morgan, A. N., Cenko, S. B., \& Perley, D. A. 2010, CBET, 2288, 1

Cobb, B. E., Bloom, J. S., Perley, D. A., Morgan, A. N., Cenko, S. B., \& Filippenko, A. V. 2010, ApJ, 718, L150

Covino, S., et al. 2008, GCN, 8331, 1

Cucchiara, A., Fox, D., Levan, A., \& Tanvir, N. 2009, GCN, 10202, 1

D'Odorico, S., et al. 2006, SPIE, 6269, 98

de Ugarte Postigo, A., et al. 2011, GCN, 11579, 1

de Ugarte Postigo, A., Thoene, C. C., \& Gorosabel, J. 2012, GCN, 12802, 1

Della Valle, M., et al. 2008, CBET, 1602, 1

Deng, J. \& Zhu, Y. 2008, GCN, 7160, 1

Djorgovski, S. G., Kulkarni, S. R., Bloom, J. S., et al. 1998, ApJ Letters, 508, L17

Evans, P. A., Page, K. L., \& Troja, E. 2009, GCN, 10201, 1

Fugazza, D., et al. 2009, GCN, 10194, 1

Fynbo, J. P. U., et al. 2006, Natur, 444, 1047

Gehrels, N., et al. 2004, ApJ, 611, 1005

Gehrels, N., et al. 2006, Natur, 444, 1044

Gelbord, J. M., et al. 2010, GCN, 11473, 1

Golenetskii, S., et al. 2009, GCN, 9553, 1

Greiner, J., et al. 2008, PASP, 120, 405

Immler, S. \& Troja, E. 2009, GCN, 10193, 1

Izzo, L., et al. 2012, arXiv, arXiv:1202.4374

Kann, D. A., Greiner, J., Kruehler, T., \& Klose, S. 2011, GCN, 12668, 1

Kuin, N. P. M. \& Gelbord, J. M. 2011, GCN, 11516, 1

Lazzarotto, F., et al. 2011, GCN, 12666, 1

Levan, A. J., Tanvir, N. R., D’Avanzo, P., Vergani, S. D., \& Malesani, D. 2010, GCN, 10523, 1

Longo, F., et al. 2009, GCN, 9524, 1

Malesani, D., et al. 2008, GCN, 7169, 1

Mazzali, P. A., et al. 2008, Sci, 321, 1185

McBreen, S. 2009, GCN, 9535, 1

Modjaz, M., et al. 2009, ApJ, 702, 226

Nishioka, Y., et al. 2009, GCN, 10224, 1

Olivares, E. F., Rossi, A., \& Greiner, J. 2010, GCN, 11478, 1

Olivares, E. F., Schady, P., Kruehler, T., Greiner, J., Klose, S., \& Kann, D. A. 2011, GCN, 11578,1

Page, K. L., et al. 2011, MNRAS, 416, 2078

Perley, D. A. 2009, GCN, 9514, 1

Raskin, C., Scannapieco, E., Rhoads, J., \& Della Valle, M. 2008, ApJ, 689, 358

Rau, A., Nardini, M., Updike, A., Filgas, R., Greiner, J., Kruehler, T., \& Klose, S. 2010, GCN, 10547,1

Roming, P. W. A., et al. 2005, Space Sci. Revs, 121, 95

Rujopakarn, W., Guver, T., Pandey, S. B., \& Yuan, F. 2009, GCN, 9515, 1

Sakamoto, T., et al. 2010, GCN, 10511, 1

Sakamoto, T., Barthelmy, S. D., Baumgartner, W. H., Cummings, J. R., Gehrels, N., Markwardt, C. B., Palmer, D. M., \& Stamatikos, M. 2010, GCN, 10524, 1

Schady, P., et al. 2009, GCN, 9512, 1

Smith, R. J., Kobayashi, S., Guidorzi, C., \& Mundell, C. G. 2009, GCN, 10192, 1

Soderberg, A. M., et al. 2008, Natur, 453, 469

Soderberg, A., Berger, E., Fox, D., Cucchiara, A., Rau, A., Ofek, E., Kasliwal, M., \& Cenko, S. B. $2008, G C N, 7165,1$

Soderberg, A., Berger, E., \& Fox, D. 2008, GCN, 8662, 1

Sparre, M., et al. 2011, ApJ, 735, L24

Stamatikos, M., et al. 2009, GCN, 10197, 1

Stamatikos, M., et al. 2010, GCN, 10496, 1

Starling, R. L. C., et al. 2011, MNRAS, 411, 2792 
Subasavage, J. P., Bailyn, C. D., Smith, R. C., Henry, T. J., Walter, F. M., \& Buxton, M. M. 2010, SPIE, 7737, 77371C

Tanaka, M., et al. 2009, ApJ, 700, 1680

Tavani, M., et al. 2009, A\& $A, 502,995$

Thoene, C. C., et al. 2009, GCN, 10233, 1

Troja, E., et al. 2009, GCN, 10191, 1

Updike, A., Rossi, A., Rau, A., Greiner, J., Afonso, P., \& Yoldas, A. 2009, GCN, 10195, 1

van der Horst, A. J. 2010, GCN, 11477, 1

Vergani, S. D., D’Avanzo, P., Levan, A. J., Covino, S., Malesani, D., Hjorth, J., \& Antonelli, L. A. 2010, GCN, 10512, 1

Vergani, S. D., Levan, A. J., D’Avanzo, P., Covino, S., Malesani, D., Hjorth, J., Tanvir, N. R., \& Antonelli, L. A. 2010, GCN, 10513, 1

Vergani, S. D., et al. 2011, GCN, 12677, 1

Wiersema, K., D’Avanzo, P., Levan, A. J., Tanvir, N. R., Malesani, D., \& Covino, S. 2010, GCN, 10525, 1

Wilson-Hodge, C. A. \& Preece, R. D. 2009, GCN, 10204, 1

Woosley, S. E. 1993, ApJ, 405, 273

Woosley, S. E. \& Bloom, J. S. 2006, ARA\&A, 44, 507

\section{Discussion}

SAVAGLIO: In your last table you have 12 GRB-SNe spectroscopically confirmed and 5 are at $z \sim 0.5$, do you have an explanation for this excess?

CoBB: There is no exact explanation for this. Of course, it is possible that this is a coincidence resulting from the small number of examples we have. Part of the problem is that most GRBs occur at much higher redshifts $(z \sim 2$ or 3$)$ and GRB-SNe are extremely difficult to detect at redshifts above $z=0.5$, due to dimness, obscuration by afterglow and host galaxy light, and the fact that the UV line-blanketed spectral regions are redshifted into the optical.

Amati: The link between long GRBs and SNe is also connected to the issue of classification of GRBs as long or short (e.g. 060614).

CoBB: Absolutely. No GRB-SN is expected following short GRBs if they are due to the merger of binary neutron stars. Therefore, if GRB 060505 and GRB 060614 are actually produced via "short-duration" progenitors, then that would naturally explain the lack of GRB-SN associated with them. In that case, they would present no challenge to the GRB-SN paradigm. Time classification is, of course, subject to issues such as detector sensitivity. Classification of bursts as short vs. long may eventually be supplanted by classification by progenitor type. 